\title{
Universality in the Random-Matrix Theory of Quantum Transport
}

\author{
C. W. J. Beenakker \\ Instituut-Lorentz, Unvversity of Leiden, P.O. Box 9506, 2300 RA Leiden, The Netherlands
}

(Received 13 November 1992)

\begin{abstract}
A random-matrix formula is derived for the variance of an arbitrary linear statistic on the transmission eigenvalues. The variance is independent of the eigenvalue density and has a universal dependence on the symmetry of the matrix ensemble. The formula generalizes the Dyson-Mehta theorem in the statistical theory of energy levels. It demonstrates that the universality of the conductance fluctuations is generic for a whole class of transport properties in mesoscopic systems.
\end{abstract}

PACS numbers: $72.10 . \mathrm{Bg}, 05.40 .+\mathrm{j}, 05.60 .+\mathrm{w}, 74.80 . \mathrm{Fp}$

In the sixties, Wigner, Dyson, Mehta, and others developed random-matrix theory (RMT) into a powerful tool to study the statistics of energy levels measured in nuclear reactions $[1,2]$. It was shown that the fluctuations in the energy level density are governed by level repulsion, which depends on the symmetry of the Hamiltonian ensemble - but is independent of the mean level density [3-6]. This universality is at the origin of the remarkable success of RMT in nuclear physics. The universality of the level fluctuations is expressed by the celebrated Dyson-Mehta formula [7] for the variance of a linear statistic $A=\sum_{n} a\left(E_{n}\right)$ on the energy levels $E_{n}$. [The quantity $A$ is called a linear statistic because products of different $E_{n}$ do not appear, but the function $a(E)$ may well depend nonlinearly on $E$.] The Dyson-Mehta formula reads

$$
\operatorname{var} A=\frac{1}{\beta} \frac{1}{\pi^{2}} \int_{0}^{\infty} d k|a(k)|^{2} k,
$$

where $a(k)=\int_{-\infty}^{\infty} d E e^{\imath k E} a(E)$ is the Fourier transform of $a(E)$, and $\beta=1,2$, or 4 depending on whether the Hamiltonian ensemble belongs to the orthogonal, unitary, or symplectic symmetry class. Equation (1) shows that (1) the variance is independent of microscopic parameters; and (2) the variance has a universal $1 / \beta$ dependence on the symmetry parameter of the ensemble.

In a seminal 1986 paper [8], Imry proposed to apply $\mathrm{RMT}$ to the phenomenon of universal conductance fluctuations (UCF), which was discovered diagrammatically by Al'tshuler [9] and Lee and Stone [10]. Shortly afterwards, a Riv $\perp$ of quantum transport was developed by Muttalib, Pichard, and Stone [11]. In this theory the role of the energy levels is played by the transmission eigenvalues $T_{n}$, or more precisely by the ratio $\lambda_{n} \equiv\left(1-T_{n}\right) / T_{n}$ of reflection and transmission coefficients. Their work is reviewed in Ref. [12], together with a closely related theory due to Mello, Pereyra, and Kumar [13]. (For still another approach, see Ref. [14].) Good agreement was obtained with the diagrammatic theory of UCF. However, it could not be shown that the universality of the fluctuations is generic for arbitrary linear statistics on the transmission eigenvalues. In particular, no formula with the generality of the Dyson-Mehta theorem could be derived. The lack of such a general theory is being felt especially now that mesoscopic fluctuations in transport properties other than the conductance (both in conductors and superconductors) have become of interest [15-17]. The obstacle which prevents a straightforward generalization of the Dyson-Mehta formula was clearly identified by Stone et al. [12]: The correlation functions in the RMT of quantum transport are not translationally invariant, due to the positivity constraint on $\lambda$. This constraint $\lambda \geq 0$ follows directly from unitarity of the scattering matrix. In contrast, the correlation functions in the RMT of energy levels are translationally invariant over the energy range of interest.

Here we wish to announce that one can overcome this obstacle towards the establishment of universality in the random-matrix theory of quantum transport.

The starting point of our analysis is the probability distribution [11]

$$
\begin{aligned}
& P\left(\left\{\lambda_{n}\right\}\right)=Z^{-1} \exp \left[-\beta \mathcal{H}\left(\left\{\lambda_{n}\right\}\right)\right], \\
& \mathcal{H}\left(\left\{\lambda_{n}\right\}\right)=-\sum_{\imath<\jmath} \ln \left|\lambda_{\imath}-\lambda_{\jmath}\right|+\sum_{\imath} V\left(\lambda_{\imath}\right),
\end{aligned}
$$

where $Z$ is a normalization constant. The variables $\lambda_{n}$ $(n=1,2, \ldots N)$ are related to the transmission eigenvalues $T_{n}$ by $T_{n}=\left(1+\lambda_{n}\right)^{-1}$. The $T_{n}$ are the eigenvalues of the matrix product $t t^{\dagger}$, where $t$ is the $N \times N$ transmission matrix of the conductor ( $N$ being the number of scattering channels). Since $\lambda$ is in one-to-one correspondence with $T$, we will call the $\lambda$ also transmission eigenvalues. As mentioned, $\lambda_{n} \geq 0$ because $0 \leq T_{n} \leq 1$. The parameter $\beta$ equals 2 if time-reversal symmetry is broken (by a magnetic field). Otherwise, $\beta$ equals 1 in the absence and 4 in the presence of strong spin-orbit scattering.

The probability distribution (2) has the form of a Gibbs distribution, with the symmetry parameter $\beta$ playing the role of inverse temperature, and the "Hamiltonian" $\mathcal{H}$ containing a logarithmic repulsive interaction plus a confining potential $V$. The function $V(\lambda)$ is chosen such that $P$ yields the required average eigenvalue density (which depends on the sample size and the degree 
of disorder). Note that $V$ may be also a function of $\beta$. The logarithmic interaction has a fundamental geometric origin: It is the Jacobian associated with the transformation from the space of scattering (or transfer) matrices to the smaller space of transmission eigenvalues [11-13]. The form (2) for the probability distribution is based on (a) an isotropy assumption, which implies that flux incident in one scattering channel is, on average, equally distributed among all outgoing channels; and (b) a maximum entropy hypothesis, which yields (2) as the least restrictive distribution consistent with a given average eigenvalue density. Assumption (a) requires a conductor much longer than wide, i.e., the quasi-one-dimensional limit. Assumption (b) has been justified by comparison with numerical simulations $[11,12]$, but there exists no rigorous proof. Indeed, it is conceivable that the true eigenvalue distribution $P\left(\left\{\lambda_{n}\right\}\right)$ cannot be fully described by a one-body potential $V(\lambda)$ plus Jacobian, as in Eq. (2), but that it contains additional many-body potentials. These would modify the logarithmic interaction of the $\lambda$. We emphasize this because one of the implications of our analysis will be that Eq. (2) is not rigorously valid - although the error is quite small.

We consider an observable $A$ which is a linear statistic on the $\lambda$, i.e.,

$$
A=\sum_{n=1}^{N} a\left(\lambda_{n}\right) .
$$

To find the variance var $A \equiv\left\langle A^{2}\right\rangle-\langle A\rangle^{2}$ we need the two-point correlation function $K_{2}\left(\lambda, \lambda^{\prime}\right)$, defined by

$$
K_{2}\left(\lambda, \lambda^{\prime}\right)=\langle\rho(\lambda)\rangle\left\langle\rho\left(\lambda^{\prime}\right)\right\rangle-\left\langle\rho(\lambda) \rho\left(\lambda^{\prime}\right)\right\rangle .
$$

Here $\rho(\lambda)=\sum_{n} \delta\left(\lambda-\lambda_{n}\right)$ is the microscopic eigenvalue density, and the brackets $\langle\cdots\rangle$ indicate the average with distribution $P\left(\left\{\lambda_{n}\right\}\right)$. The mean density is

$$
\langle\rho(\lambda)\rangle=\frac{\int d \lambda_{1} \cdots \int d \lambda_{N} \rho(\lambda) \exp (-\beta \mathcal{H})}{\int d \lambda_{1} \cdots \int d \lambda_{N} \exp (-\beta \mathcal{H})} .
$$

Once the correlation function $K_{2}$ is known, the variance of the linear statistic (3) follows from

$$
\operatorname{var} A=-\int_{0}^{\infty} d \lambda \int_{0}^{\infty} d \lambda^{\prime} a(\lambda) a\left(\lambda^{\prime}\right) K_{2}\left(\lambda, \lambda^{\prime}\right) .
$$

Our method is to relate the correlation function to a functional derivative of the eigenvalue density with respect to $V$, and then to evaluate this functional derivative in the limit $N \rightarrow \infty$. A similar line of reasoning was used by Politzer [18], for a different purpose (viz., to show that $A$ has a Gaussian distribution). We discuss the two steps of our method separately.

(1) The functional derivative of $\langle\rho(\lambda)\rangle$ with respect to $V\left(\lambda^{\prime}\right)$ consists of two terms: Differentiation of the numerator in Eq. (5) gives $-\beta\left\langle\rho(\lambda) \rho\left(\lambda^{\prime}\right)\right\rangle$, since $\delta \mathcal{H} / \delta V(\lambda)=\rho(\lambda)$. Differentiation of the denominator gives $\beta\langle\rho(\lambda)\rangle\left\langle\rho\left(\lambda^{\prime}\right)\right\rangle$. The two terms together yield

$$
K_{2}\left(\lambda, \lambda^{\prime}\right)=\frac{1}{\beta} \frac{\delta\langle\rho(\lambda)\rangle}{\delta V\left(\lambda^{\prime}\right)} .
$$

Substitution into Eq. (6) then gives

$$
\operatorname{var} A=-\frac{1}{\beta} \int_{0}^{\infty} d \lambda \int_{0}^{\infty} d \lambda^{\prime} a(\lambda) a\left(\lambda^{\prime}\right) \frac{\delta\langle\rho(\lambda)\rangle}{\delta V\left(\lambda^{\prime}\right)} .
$$

This relationship between the variance of a linear statistic and the functional derivative of the density of transmission eigenvalues is an exact consequence of the probability distribution (2).

(2) To evaluate the functional derivative (7) we must know how the density of transmission eigenvalues $\langle\rho\rangle$ depends on the potential $V$ in the Hamiltonian (2). There exists a one-to-one relationship between these two quantities, because $V$ is assumed to be a one-body potential. For large $N$ the relationship is given by the integral equation $[4,19]$

$$
\int_{0}^{\infty} d \lambda^{\prime}\left\langle\rho\left(\lambda^{\prime}\right)\right\rangle \ln \left|\lambda-\lambda^{\prime}\right|+\frac{\beta-2}{2 \beta} \ln \langle\rho\rangle=V+c .
$$

The constant $c$ is to be determined from the normalization condition $\int d \lambda\langle\rho\rangle=N$. The second term on the left-hand side of Eq. (9) is of order $N^{-1} \ln N$ relative to the first, and terms of still higher order in $1 / N$ are neglected. To calculate the two-point correlation function (7) in leading order it is sufficient to retain only the first term, so that we can work with the linear integral equation

$$
\int_{0}^{\infty} d \lambda^{\prime}\left\langle\rho\left(\lambda^{\prime}\right)\right\rangle \ln \left|\lambda-\lambda^{\prime}\right|=V(\lambda)+c .
$$

Equation (10) has the intuitive "mean-field" interpretation (originally due to Wigner) that the "charge density" $\langle\rho\rangle$ adjusts itself to the "external potential" $V$ in such a way that the total force on any charge $\lambda$ vanishes [12]. The more accurate Eq. (9) shows that, in fact, Eq. (10) is the leading term in a $1 / N$ expansion.

Equations (8) and (10) have two immediate implications for the universality of the variance of a linear statistic on the transmission eigenvalues: (1) Equation (10) is a linear relation between $\langle\rho\rangle$ and $V$, and hence the functional derivative $\delta\langle\rho\rangle / \delta V$ is independent of $V$. Since all microscopic parameters enter via the potential $V(\lambda)$, this implies that the variance (8) is independent of microscopic parameters. (2) The kernel in Eq. (10) does not contain $\beta$, and hence the variance (8) has a universal $1 / \beta$ dependence on the symmetry parameter. This conclusion holds irrespective of any $\beta$ dependence of $V(\lambda)$. If one is only interested in the universality of the mesoscopic fluctuations, one can stop here. To calculate the numerical value of var $A$ requires a little more work. The integral equation (10) can be solved by Mellin transformation, which yields the functional derivative $\delta\langle\rho\rangle / \delta \mathrm{V}$ as the solving kernel. Here we only give the results; mathematical details of the calculation will be published elsewhere [20]. 
The two-point correlation function $K_{2}\left(\lambda, \lambda^{\prime}\right)$ which follows from Eqs. (7) and (10) is

$$
K_{2}\left(\lambda, \lambda^{\prime}\right)=\frac{1}{\pi^{2} \beta} \frac{\partial}{\partial \lambda} \frac{\partial}{\partial \lambda^{\prime}} \ln \left|\frac{\sqrt{ } \lambda-\sqrt{ } \lambda^{\prime}}{\sqrt{ } \lambda+\sqrt{ } \lambda^{\prime}}\right| .
$$

This function is obviously not translationally invariant. However, a translationally invariant kernel can be obtained by the transformation $\lambda=e^{x}, \tilde{K}_{2}\left(x, x^{\prime}\right)=$ $e^{x+x^{\prime}} K_{2}\left(e^{x}, e^{x^{\prime}}\right)$. The result is

$$
\tilde{K}_{2}\left(x, x^{\prime}\right)=-\frac{1}{\pi^{2} \beta} \frac{d^{2}}{d\left(x-x^{\prime}\right)^{2}} \ln \left|\tanh \frac{x-x^{\prime}}{4}\right| .
$$

Substituting Eq. (11) into Eq. (6), and carrying out two partial integrations, we find the formula [21]

$$
\begin{aligned}
\operatorname{var} A=-\frac{1}{\beta} \frac{1}{\pi^{2}} \int_{0}^{\infty} d \lambda \int_{0}^{\infty} & d \lambda^{\prime} \ln \left|\frac{\sqrt{ } \lambda-\sqrt{ } \lambda^{\prime}}{\sqrt{ } \lambda+\sqrt{ } \lambda^{\prime}}\right| \\
& \times \frac{d a(\lambda)}{d \lambda} \frac{d a\left(\lambda^{\prime}\right)}{d \lambda^{\prime}}
\end{aligned}
$$

In an equivalent Fourier representation, we can write

$$
\operatorname{var} A=\frac{1}{\beta} \frac{1}{\pi^{2}} \int_{0}^{\infty} d k|\tilde{a}(k)|^{2} k \tanh (\pi k) .
$$

Here $\tilde{a}(k)$ is the Mellin transform of $a(\lambda)$, i.e., the Fourier transform with respect to $x \equiv \ln \lambda$ :

$$
\tilde{a}(k)=\int_{0}^{\infty} d \lambda \lambda^{\imath k-1} a(\lambda)=\int_{-\infty}^{\infty} d x e^{\imath k x} a\left(e^{x}\right) .
$$

The kernel in Eq. (14) is the Fourier transform with respect to $x-x^{\prime}$ of $\tilde{K}_{2}\left(x, x^{\prime}\right)$. Equation (14) is for the quantum transport problem what the Dyson-Mehta formula (1) was for the statistics of energy levels.

As an independent check of the validity of our key result, we have compared Eq. (14) with an exactly solvable model. This is the Laguerre ensemble, defined by Eq. (2) with $\beta=2$ and $V(\lambda)=\frac{1}{2} \lambda-\frac{1}{2} \alpha \ln \lambda$. The parameter $\alpha>-1$ is arbitrary. The correlation function for this ensemble is known exactly, in terms of generalized Laguerre polynomials $[12,22]$. In Fig. 1 we show the comparison for the variance of the conductance. The conductance $G$ is a linear statistic on the transmission eigenvalues, according to the Landauer formula

$$
G / G_{0}=\sum_{n} T_{n}=\sum_{n} \frac{1}{1+\lambda_{n}}
$$

Here $G_{0}=2 e^{2} / h$ is the conductance quantum. The Mellin transform of $a(\lambda)=(1+\lambda)^{-1}$ is $\tilde{a}(k)=$ $-i \pi / \sinh (\pi k)$. Substitution into Eq. (14) yields the variance

$$
\operatorname{var}\left(G / G_{0}\right)=\beta^{-1} \int_{0}^{\infty} d k \frac{2 k}{\sinh (2 \pi k)}=\frac{1}{8} \beta^{-1} .
$$

For the Laguerre ensemble (which has $\beta=2$ ) we would thus expect from our variance formula that $\operatorname{var}\left(G / G_{0}\right)=$ 0.0625 for $N \gg 1$, independent of $N$ and of the parameter $\alpha$ (which in this model plays the role of a "microscopic"

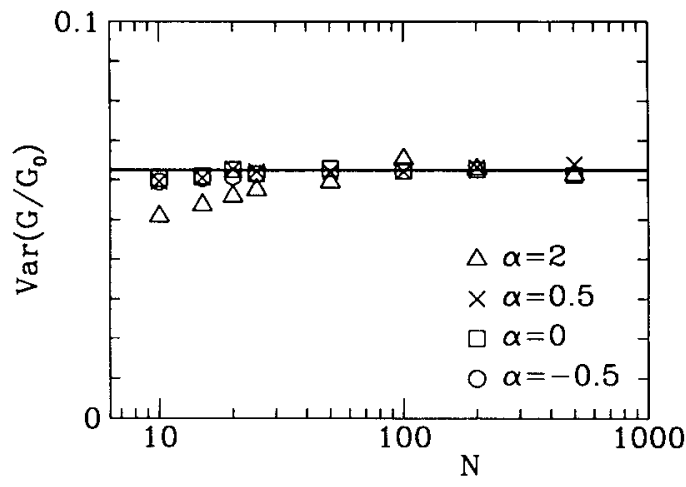

FIG. 1. Variance of the conductance $G$ (in units of $G_{0}=$ $\left.2 e^{2} / h\right)$ as a function of the number of channels $N$. The data points are obtained by integration of the exact correlation function for the generalized Laguerre ensemble $[12,22]$, for various values of the microscopic parameter $\alpha$. The estimated error in the numerical integration is \pm 0.001 . (For $\alpha=-0.5$ we could only integrate with the required accuracy for $N$ up to 25.) The horizontal line at 0.0625 is the $\alpha$ independent value predicted in the limit $N \rightarrow \infty$ by Eq. (14).

parameter). As one can see in Fig. 1, this is indeed what we find (within numerical accuracy) from integration of the exact correlation function.

The coefficient $\frac{1}{8}$ in Eq. (17) is close to but not precisely identical to the established value $\frac{2}{15}$ for a quasi-onedimensional conductor $[10,23]$. The smallness of the difference explains why it was not noticed previously. From a practical point of view, the difference is not really significant, but conceptually it has the important implication that the RMT based on the probability distribution (2) is not rigorously equivalent to the diagrammatic theory of UCF [24], which we hold to be exact. The conclusion is that the interaction between the $\lambda$ is not precisely logarithmic.

The variance formula (14) can be readily applied to other transport properties which are linear statistics. As an illustration, we briefly discuss some examples which have previously been studied by other methods [15-17]. The first example is the shot-noise power $P$ of a phasecoherent conductor, given by [25]

$$
P / P_{0}=\sum_{n} T_{n}\left(1-T_{n}\right)=\sum_{n} \frac{\lambda_{n}}{\left(1+\lambda_{n}\right)^{2}} .
$$

Here $P_{0}=2 e|U| G_{0}$, with $U$ the applied voltage. The variance formula (14) yields $\operatorname{var}\left(P / P_{0}\right)=\frac{1}{64} \beta^{-1}$. The second example is the conductance $G_{\mathrm{NS}}$ of a disordered microbridge between a normal $(\mathrm{N})$ and a superconducting $(\mathrm{S})$ reservoir, which is related to the transmission eigenvalues in the normal state by [26]

$G_{\mathrm{NS}} / G_{0}=2 \sum_{n}\left(\frac{T_{n}}{2-T_{n}}\right)^{2}=\sum_{n} \frac{2}{\left(1+2 \lambda_{n}\right)^{2}}$.

This expression holds only in zero magnetic field and for spin-independent scattering, i.e., for $\beta=1$. Applying 
Eq. (14) we obtain $\operatorname{var}\left(G_{\mathrm{NS}} / G_{0}\right)=\frac{9}{16}$, where we have set $\beta$ equal to 1 . For broken time-reversal symmetry the conductance of the NS junction is not a linear statistic $[26]$; hence no $1 / \beta$ dependence of the variance is to be expected. The third example is the supercurrent phase relationship $I(\phi)$ of a point-contact Josephson junction, which for $\beta=1$ is given by [15]

$$
I(\phi)=\frac{e \Delta}{2 \hbar} \sum_{n} \frac{T_{n} \sin \phi}{\left[1-T_{n} \sin ^{2}(\phi / 2)\right]^{1 / 2}},
$$

$\Delta$ being the energy gap in the bulk superconductor. Application of the variance formula (14) to the linear statistic (20) yields a rms value which increases linearly at small $\phi$ and saturates at $\operatorname{rms} I(\pi)=\pi^{-1} e \Delta / \hbar$ as $\phi$ approaches $\pi$. For the critical current $I_{c} \equiv \max I(\phi)$ we find [27] $\mathrm{rms} I_{c}=0.29 \mathrm{e} \Delta / \hbar$.

We have checked for all these transport properties that the variances predicted by Eq. (14) agree with the numerical results from the Laguerre ensemble.

In conclusion, we have derived the analog of the DysonMehta theorem [7] for the quantum transport problem. The formula obtained demonstrates that the universality which was the hallmark of the phenomenon of "universal conductance fluctuations" $[9,10]$ is generic for linear statistics on the transmission eigenvalues. This universality was anticipated [8] from the random-matrix theory of energy levels, but could not previously be established because of the absence of translational invariance of the correlation function of transmission coefficients (originating from the unitarity of the scattering matrix) [12]. Finally, our analysis has revealed a small but real numerical discrepancy between the random-matrix theory [11] and the diagrammatic calculation $[9,10]$, which implies that the interaction between the $\lambda$ eigenvalues is not precisely logarithmic.

I have benefited from discussions with $\mathrm{R}$. A. Jalabert, M. J. M de Jong, J. M. J. van Leeuwen, B. Rejaei, and A. D. Stone. I thank R. A. Jalabert for helping me with the numerical integrations. This research was supported by the Dutch Science Foundation NWO/FOM.

(1) M. L. Mehta, Random Matrices (Academic, New York, 1991), 2nd ed.

[2] T. A. Brody, J. Flores, J. B. French, P. A. Mello, A. Pandey, and S. S. M. Wong, Rev. Mod. Phys. 53, 385 (1981).

[3] D. Fox and P. B. Kahn, Phys. Rev. 134, B1151 (1964).

[4] F. J. Dyson, J. Math. Phys. 13, 90 (1972).

[5] A. Pandey, Ann. Phys. (N.Y.) 134, 110 (1981).

6] R. D. Kamien, H. D. Politzer, and M. B. Wise, Phys. Rev. Lett. 60, 1995 (1988).

[7] F. J. Dyson and M. L. Mehta, J. Math. Phys. 4, 701 (1963).
[8] Y. Imry, Europhys. Lett. 1, 249 (1986).

[9] B. L. Al'tshuler, Pis'ma Zh. Eksp. Teor. Fiz. 41, 530 (1985) [JETP Lett. 41, 648 (1985)].

[10] P. A. Lee and A. D. Stone, Phys. Rev. Lett. 55, 1622 (1985).

[11] K. A. Muttalib, J.-L. Pichard, and A. D. Stone, Phys. Rev. Lett. 59, 2475 (1987).

[12] A. D. Stone, P. A. Mello, K. A. Muttalib, and J.-L. Pichard, in Mesoscopic Phenomena in Solids, edited by B. L. Al'tshuler, P. A. Lee, and R. A. Webb (NorthHolland, Amsterdam, 1991).

[13] P. A. Mello, P. Pereyra, and N. Kumar, Ann. Phys. (N.Y.) 181, 290 (1988).

[14] B. L. Al'tshuler and B. I. Shklovskiř, Zh. Eksp. Teor. Fiz. 91, 220 (1986) [Sov. Phys. JETP 64, 127 (1986)].

[15] C. W. J. Beenakker, Phys. Rev. Lett. 67, 3836 (1991); 68, 1442(E) (1992).

[16] Y. Takane and H. Ebisawa, J. Phys. Soc. Jpn. 60, 3130 (1991); 61, 2858 (1992).

[17] M. J. M. de Jong and C. W. J. Beenakker, Phys. Rev. B 46, 13400 (1992).

[18] H. D. Politzer, Phys. Rev. B 40, 11917 (1989).

[19] In Dyson's derivation [4] of Eq. (9), all integrals run from $-\infty$ to $+\infty$. In our case, the integration range is from 0 to $\infty$. We have checked that this positivity constraint on $\lambda$ does not introduce any extra terms in the integral equation, to the order considered.

[20] C. W. J. Beenakker (unpublished).

[21] Equation (13) requires that $a(\lambda)$ be differentiable. In particular, var $A$ diverges logarithmically for a step function, $a(\lambda)=\theta\left(\lambda_{c}-\lambda\right)$. For such artificial linear statistics the variance does not have a universal $N \rightarrow \infty$ limit, but increases as $\ln N$ for large $N$ [7]. All physical properties, however, are smooth (differentiable) functions of $\lambda$.

[22] K. Slevin, J.-L. Pichard, and P. A. Mello, Europhys. Lett. 16, 649 (1991). The "two-level cluster function" $T_{2}$ considered in this reference is related to the "twopoint correlation function" $K_{2}$ of the present paper by $K_{2}\left(\lambda, \lambda^{\prime}\right)=T_{2}\left(\lambda, \lambda^{\prime}\right)-\langle\rho(\lambda)\rangle \delta\left(\lambda-\lambda^{\prime}\right)$.

[23] P. A. Mello, Phys. Rev. Lett. 60, 1089 (1988); P. A. Mello and A. D. Stone, Phys. Rev. B 44, 3559 (1991).

[24] A second implication of $\frac{1}{8} \neq \frac{2}{15}$ is that the RMT of Muttalib, Pichard, and Stone [11] (the so-called "global approach") is not precisely equivalent to the "local approacil" of Mello et al. [13, 23]. Previous work by Mello and Pichard [Phys. Rev. B 40, 5276 (1989)] argues for the equivalence of the two theories. Their argument starts from a one-body potential $V(\lambda)$, i.e., it assumes that the interaction between the $\lambda$ is precisely logarithmic. We now know that this is an approximation (albeit an excellent one).

[25] M. Büttiker, Phys. Rev. Lett. 65, 2901 (1990).

[26] C. W. J. Beenakker, Phys. Rev. B 46, 12841 (1992).

[27] We have used that for $N \rightarrow \infty$, var $I_{c}=\operatorname{var} I\left(\phi_{c}\right)$, where $\phi_{c}=1.97$ is the phase difference at which the ensemble average $\langle I(\phi)\rangle$ reaches its maximum. See C. W. J. Beenakker, in Transport Phenomena in Mesoscopic Systems, edited by H. Fukuyama and T. Ando (Springer, Berlin, 1992). 